\title{
The Comparative Study on English and Chinese Intonation
}

\author{
Qi Pan \\ English Department, Zhenjiang Watercraft College of PLA, Zhenjiang, China \\ Email: panqi_tracy@126.com
}

\begin{abstract}
Intonation, being the essential element and external form of a language, serves the human beings primarily as a medium of communication. The contrastive analysis of English and Chinese intonation has great significance in the development of contrastive phonology and improvement of foreign language learning and teaching practice. This article attempts to make a contrastive study of English and Chinese intonation from two aspects: structure and function. The differences and similarities between English and Chinese intonation can clarify some misunderstandings in both English and Chinese intonation teaching.
\end{abstract}

Index Terms - intonation, contrastive analysis of English and Chinese, structure, function

\section{INTRODUCTION}

Intonation has long been considered as an important linguist phenomenon in verbal communication, where it commonly serves the function of enabling words to convey the intended meaning. Halliday once mentioned: 'We can not fully describe the grammar of spoken English without reference to contrasts expounded by intonation.' It is not what we said; it is how we said it. In foreign language learning, the special significance of intonation is even more outstanding. It is quite common that what most easily and reliably distinguish an advanced foreign language learner from the native speakers is often not his words or expressions but his intonational performance.

Intonation is not an area of language to which much attention was paid before the twentieth century. So far the three most influential approaches to English intonation are the configurational approach of the British tradition, the level approach of the American tradition, and most recently the AM approach in the generative framework. Within a history of up to four or five hundred years, a huge volume of literature in each approach has been produced. Compared with English, Chinese is a typical tone contour language. In Chinese, tone and intonation co- exist in the intonation F0 curves. Among them, tone is lexically specified. Therefore, a great many questions will arise regarding how Chinese intonation is actually formed. A great number of theories and models concerning Chinese intonation have been proposed so far since the late twentieth of the last century.

As for the contrastive study of English and Chinese intonation, the study started in 1933 when Yuan-Ren Chao published his article "a Preliminary Study of English Intonation (with American Variants) and its Chinese Equivalent". In Britain, Palmer divided English intonation into three parts: head, nucleus and tail. Based on Palmer's understanding and for the convenience of comparison with Chinese intonation, Chao further analyzed Chinese tone group into "Head" and "Body". "Head" includes anacrusis and main head. "Anacrusis" refers to the unstressed syllables before the first stressed syllable in the head and the remaining part in it is called "Main Head". "Body" is composed of nucleus and tail.

\section{A COMPARISON OF THE STRUCTURES OF ENGLISH AND CHINESE INTONATION}

\section{A. English Intonation Structure}

The unit of intonation in English is the tone unit. Other names for it include tone-group, sense-group, intonation-group, intonational phrase and intonation contour.

In the light of intonation theory, a single English utterance or sentence may be considered as a tone unit or a combination of two or more tone units, if it can be said with a complete tone-pattern and conveys a comparatively complete meaning. In many cases, in conversational English, there is a tendency for the tone units to correspond to sense groups or major grammatical chunks, and this can be taken as the basic unmarked pattern: neutral in tonality. However, at the same time there is no reliable relationship between a tone unit and "sentence" or "clause" or any other kind of grammatical item, because the tone unit is a meaningful unit in its own right. The tone unit is one unit of information in the message that the speaker is communicating and can be of any length. The particular meaning the speaker wishes to convey under a particular context may make him feel it necessary to break a single clause into two or more tone units, or to combine two or more clauses into one single tone unit.

Tone units enable the listener to isolate pitch patterns by defining where one pattern ends and the next begins and help the listener understand messages. Therefore it is important to identify tone units in the communication. A few general rules on dividing a message into tone units on phonetic grounds are as follows:

a. Pause 
This is the most frequently used criterion in tone unit identification. A pause, long or short, may signal a major boundary, principally between clauses or between the subject and the predicate. During the pause the speaker can get a fresh breath for the following tone unit. But we can detect some other criteria as oral indicators of tone unit boundaries. The main points below are based on Cruttenden.

b. Pre-head

The presence of the unaccented pre-head generally indicates the beginning of a tone unit, for example: "I saw John yesterday/and he was just off to London.//",the most likely place for the onset in the second tone unit is on "just" and the unaccented syllables in the pre-head are like to be uttered more quickly than those elsewhere in the sentence (and, specifically, more quickly than those at the end of yesterday). The sudden acceleration beginning at "and" indicates that these three syllables in the pre-head i.e. "and he was", are anacrustic and that they mark the beginning of a new tone unit.

c. Tail

The nuclear syllable, as well as those in the tail of a tone unit, will often be lengthened. In the above example, both "yesterday" and "London" are lengthened suggesting a tone unit boundary.

d. Pitch

This criterion concerns the pitch of unaccented syllables. A change in pitch level and/ or pitch direction among unaccented syllables in the tail of the previous tone unit and those in the pre-head of the following tone unit signals generally a tone unit boundary.After the falling tone's low tail syllables, there will be a slight step-up to the pitch level of the pre-head of a new tone unit. Following rising tones, a step-down can be heard to the pitch level of any unaccented syllable of the pre-head of the next tone unit.

e. Nucleus

Finally, and most importantly a tone unit must contain at least a nuclear syllable or nucleus with pitch accent. Pauses do not always mark tone unit boundaries. A word (group) separated from the rest of the sentence by a pause but containing no such a syllable at all does not constitute a tone unit.

\section{B. Chinese Intonation Structure}

The opinions diverge on the unit of Chinese intonation. Some introduce the term sentence intonation and investigate Chinese intonation at sentence level while the others refer to the notion of intonation-group and separate some prosodic units on the "defining features" they believe.

And some other sentence intonation holders did not point this out directly, though all of their study units accorded with sentence. For example, Yuan-Ren Chao proposed "algebraic sum" theory, holding that the tones are integrated with intonation just like small ripples riding on large waves, the relationship between which is a kind of "algebraic sum", but his attention was mainly concentrated on the last syllable of a phrase or a sentence, while ignored other parts of the utterance. Prosodic units holders are mainly the scholars in the Institute of Linguistics, who try to define the minimal intonation units based on tone interaction or some symbols occurring on the F0 curves.

But this divergence may ultimately turn out to be a trivial question, since they are barely different methods to analyze intonation and do not exclude each other from the root. To a certain extent, they may reconcile with each other, i.e. a state of coexistence. For example, they all admit that pitch accent and boundary tone at sentence-final are essential to Chinese intonation. Since the patterns of Chinese intonation mainly occur repeatedly at the sentence level, it is appropriate to take sentence as the carrier for Chinese intonation.

\section{Contrastive Analysis of Intonation Structures of English and Chinese}

\section{a. Similarities}

1. The components of intonation structure are similar in both languages, including four parts: pre-head, head, nucleus and tail. Nucleus is essential in intonation structure, which is usually perceived as the most prominent part in the sentence and plays the most important part in showing intonation information. Pre-head is at the beginning of a sentence and usually contains a few weak syllables. The function of pre-head in both languages is also similar. Both languages have two kinds of pre-head: low pre-head and high pre-head and use pre-head as the modifier of intonation meanings. Low pre-head is usually regarded as normal pre-head and adds no meaning to the tone, however, high pre-head is usually used to add vivacity, liveliness, excitement and vehemence to whatever other attitudes the nucleus expresses.

2. The intonation patterns are similar in both languages, including four kinds: rising, falling, fluctuation and level. Some of the intonation patterns also have the similar meanings. The falling intonation in both languages can be used to show definiteness, completeness, and command. The rising intonation can be used to show question and doubt. The level intonation can be used to show something normal and routine. The fluctuational intonation can be used to show complicated moods and implying meanings.

b. Differences

1. The unit of intonation is different. The unit of intonation in English is tone unit. An English sentence is composed by one or more tone units. Each of them has a nucleus and then a nuclear tone. The unit of intonation in Chinese is often referred to as a sentence and a Chinese sentence is often marked by a boundary tone at the sentence final. This difference impresses us that an English sentence seems to involve more pitch movements for intonation purpose than the Chinese one and in reverse, the main body of a Chinese sentence seems to be level though it may be ended by a 
remarkable boundary tone.

2. The components of intonation structure play different parts in forming intonation patterns. In English, nucleus carries the main burden of the pitch movement in the tone unit. It covers the widest pitch range, so if, for example, the tone unit is on a falling tone the nucleus will have a greater falling movement than any of the other syllables. It will fall more steeply and over a wider range. The nucleus is the most important part of a tone unit because it expresses the speaker's feeling, mood, attitude, emotion and the meaning he wishes to express .Nucleus is also essential in Chinese intonation, however, the change of intonation height is often shown in the sentence final, especially in rising and falling intonation. So the intonation patterns are often shown in the tail, sometimes in the nucleus, even in the pre-head. Because of this difference, Chinese students usually pay more attention to the tail when forming English intonation patterns and don't get used to raising or falling the intonation from the nucleus and spreading over the tail. Therefore, helping Chinese student to master the correct way to pronounce nuclear tone in English and remove Chinese negative transfer is our focus in the teaching of English pronunciation.

\section{A COMPARISON OF THE FUNCTION OF ENGLISH AND CHINESE INTONATION}

Intonation is an essential component in language communication. Spoken language consists not only of grammatical forms, but also communicates complex layers of person to person emotions, cognitive and hierarchical organization of topics, and cooperative signals to indicate degree of understanding and the success of the communication. Like the other branches of linguistics, the phonological study is not only involved in the linguistic structures, but also in their functions. After all, language is the primary means of communication, whereas communication is the main function of language. Consequently, the author attempts to make a contrastive analysis between English and Chinese intonation from the functional perspective in the following section.

It has been established that intonation helps the speakers to convey information effectively. When we talk to someone, we don't just exchange ideas, we interact with them - there is a social relationship between the speaker and the listener. To hold a conversation, we need to know not only the language for the functions we want to express, but also the rules for interacting - when we can speak, when we can interrupt, how we can check what the other person has said, how we can connect our speech with what has gone before, and many others. To put it in the broadest possible terms, we can see that intonation, which is one part of the structure of interaction, makes it easier for a listener to understand what a speaker is trying to convey. For this reason, we shall investigate in this chapter what functions intonation can exercise.

Descriptions on the functions of intonation may vary, but what we are concerned with is whatever helps us to facilitate learning and communication. Ideally, we shall focus on the functions that are regarded as most important. These may include attitudinal function, discoursal function, grammatical function and accentual function.

\section{A. The Attitudinal Function of Intonation}

In 1968, Cook has pointed out, “A mistake in intonation like a grammatical mistake is a mistake in one's use of the language. However, an Englishman who hears a mistake in intonation does not react in the same way as when he hears a grammatical mistake; he is prepared to make allowances for the foreigner's faulty grammar but he does not make the same allowances for the foreigner's intonation. Instead he will think, not that the foreigner is making a mistake, but that he possesses the wrong attitude." Cook attaches great importance to the attitudinal function of intonation. Intonation enables us to express emotions and attitudes when we speak, which adds a special kind of meaning to spoken language. This is often called the attitudinal function of intonation. One sentence spoken in different ways can be labeled by different moods or attitudes, such as happy, sad, excited, angry, etc. For example, most English books agree on some basic attitudinal meanings of tunes: fall means finality, definiteness; rise means general questions, listing, more to follow and encouraging; fall-rise means uncertainty, doubt; rise-fall means surprise, being impressed. And the carriers of this function in English intonation mainly are width of pitch range, key, loudness, speed, and voice quality. (Peter Roach, 2000)

\section{B. The Discoursal Function of Intonation}

This is a comparatively new area of study in the description of natural speech. Not long ago linguistic analysis is usually linked to the sentence as the maximum unit of grammar. But the study of discourse attempts to look at the larger contexts in which sentences occur. Looking at the way intonation is used in specific situations highlights the crucial role that context plays in determining what people say and how they say things. The way an interaction develops will depend heavily on such factors as who is involved in it and what the relation is between the speakers, the topic of the conversation and how much the participants know about the details of the topic and so on. One role of intonation is to help lubricate and regulate this interaction by signaling certain features of discourse.

\section{The Grammatical Function of Intonation}

"The listener is better able to recognize the grammar and syntactic structure of what is being said by using the information contained in the intonation: for example, such things as the placement of boundaries between phrases, clauses or sentences, the difference between questions and statements and the use of grammatical subordination may be indicated. This has been called the grammatical function of intonation.”(Peter Roach, 2000, p.109). 


\section{The Accentual Function of Intonation}

"Intonation helps to produce the effect of prominence on syllables that need to be perceived as stressed, and in particular the placing of tonic stress on a particular syllable marks out the word to which it belongs as the most important in the tone-unit. This has been called the accentual function of intonation."(Peter Roach, 2000, p.75)It implies that the nucleus is the most important part in a tone unit and the placement of nucleus can indicate different information focus in the discourse. Therefore, the accentual function of intonation can be understood as the focus function.

\section{CONCLUSION}

Intonation, being the essential element and external form of a language, serves the human beings primarily as a medium of communication. Contrastive analysis is always recognized as an effective approach for language learning and teaching, especially on phonological level. Therefore, the contrastive analysis of English and Chinese intonation has great significance in foreign language learning and teaching field.

A successful comparison of Chinese and English intonation is obviously of great significance in both theoretical and practical areas. In the process of comparing, some "vague" features of intonation in one language are clarified with the reference to some "prominent" features in another language. And another, some researching notions and means on the intonation study of one language may facilitate the study in another language. Besides this, the practical significance of the comparison is especially remarkable. It will be of a big help to not only the English learners in China but also the English natives who are learning English. Both of them should overcome the transition of the intonation patterns of their mother tongue into the target language, and what's more, the strong influence of tone-pattern in Chinese should not be neglected either in their studies.

In this article, I made a contrastive analysis of the intonation system in English and Chinese from two aspects: the structure and function. After the comparison, the similarities and differences between the intonation systems of the two languages are easy to recognize. My article attempts to realize the significance and apply the study to foreign or second language learning and teaching.

\section{REFERENCES}

[1] Anne Wichmann. (2000). Intonation in Text and Discourse.Singapore: Longman.

[2] Cook. (1968). Active Intonation. Singapore: Longman.

[3] Halliday, M.A.K. (1970). A Course of Spoken English: Intonation. Oxford: Oxford University Press.

[4] Ladd, D. (1996). Intonational Phonology. Cambridge: Cambridge University Press.

[5] Peter Roach. (2000). English Phonetics and Phonology: A Practical Course. Beijing: Foreign Language Teaching and Research Press.

Qi Pan was born in Zhenjiang, China in 1987. She received her B.A. degree in English language and literature from Jiangsu University of Science and Technology, China in 2009.

She is currently a tutor in English Department, Zhenjiang Watercraft College of PLA, Zhenjiang, China. Her research interests include Second Language Acquisition Contrastive Studies of English and Chinese. 